\title{
Video-assisted thoracic surgery pneumonectomy: the first case report in Poland
}

\author{
Cezary Piwkowski, Piotr Gabryel, Mariusz Kasprzyk, Wojciech Dyszkiewicz \\ Thoracic Surgery Department, Poznan University of Medical Sciences, Poland
}

Videosurgery Miniinv 2012; 7 (3): 197-201 DOI: 10.5114/wiitm.2011.28869

\begin{abstract}
Minimally invasive video-assisted thoracic surgery (VATS) lobectomy has proved to be equal and in some aspects superior to open lobectomy in T1 and T2 lung cancers. Indications for VATS pneumonectomy however are still not clearly defined and strictly limited. The minimally invasive VATS pneumonectomy can be undertaken in patients with centrally located tumors without extended invasion of the large pulmonary vessels, chest wall, pericardium, mediastinal structures or proximal part of the main bronchus and when sleeve resection is not feasible (T2). We present a case of a patient who underwent left VATS pneumonectomy due to left lung cancer. Based on the preoperative examinations any kind of less extensive resection than pneumonectomy was excluded. Our VATS technique consisted of three incisions: two ports and a 5-6 cm long utility incision without any kind of rib spreading. The whole of the procedure was controlled on the monitor via a thoracoscope and the technique of resection was described in detail. Total surgery time was 130 min and blood loss was $150 \mathrm{ml}$. The chest tube was removed on the $2^{\text {nd }}$ post-operative day and the patient was discharged home on the $8^{\text {th }}$ postoperative day. The final histopathological examination confirmed squamous cell lung cancer (T2aNOMO stage IB). In the authors' opinion VATS pneumonectomy should be performed only in centers with extensive experience in minimally invasive VATS lobectomy. Despite limited indications for VATS pneumonectomy, if the patients fulfill the VATS pneumonectomy inclusion criteria they may gain from all the advantages of minimally invasive techniques.
\end{abstract}

Key words: video-assisted thoracic surgery, pneumonectomy, lung cancer.

\section{Introduction}

Minimally invasive video-assisted thoracic surgery (VATS) lobectomy has proved to be equal and in some aspects superior to open lobectomy in $\mathrm{T} 1$ and T2 lung cancers. Its advantages compared to thoracotomy are less surgical injury, decreased postoperative pain, fewer postoperative pulmonary complications, shorter hospital stay, improved quality of life, and improved delivery of adjuvant chemotherapy, with a comparable long-term survival rate [1-3]. The rate of thoracoscopic lobectomies is steadily increasing and in leading thoracic centers has already exceeded $50 \%$ of all resections. Indications for VATS pneumonectomy however are still not clearly defined and strictly limited. This results in a low number of cases operated on each year $[4,5]$.

We present a case of a patient who underwent left VATS pneumonectomy due to lung cancer at the Thoracic Surgery Department of Poznan University of Medical Sciences with special consideration of technical aspects of the procedure.

\section{Case report}

The 63-year old patient with left lung cancer was admitted to the Thoracic Surgery Department. On admission the patient gave a 3-month history of dry

Address for correspondence:

Cezary Piwkowski MD, PhD, Thoracic Surgery Department, Poznan University of Medical Sciences, 62 Szamarzewskiego, 60-569 Poznan, Poland, phone: +48616654358, e-mail: cezary_p@hotmail.com 
cough, short breath on exertion and $10 \mathrm{~kg}$ weight loss. He had a history of 43 pack years of smoking. He stopped smoking two weeks before the surgery. The main comorbidities were arterial hypertension and chronic obstructive lung disease. On physical examination breath sounds were slightly decreased over the left lung. Chest X-ray showed enlargement of the left hilum. Computed tomography (CT) scan of the chest revealed a $4.2 \mathrm{~cm}$ mass located in the distal portion of the left main bronchus without infiltration of the pulmonary vessels in the hilum and mediastinum. The left main bronchus was almost completely obstructed and the lung parenchyma overinflated. No enlargement of the mediastinal lymph nodes was observed. Bronchoscopy confirmed exophytic tumor obliterating the distal part of the left main bronchus and infiltrating orifices of both the upper and the lower lobar bronchi. A biopsy was car-

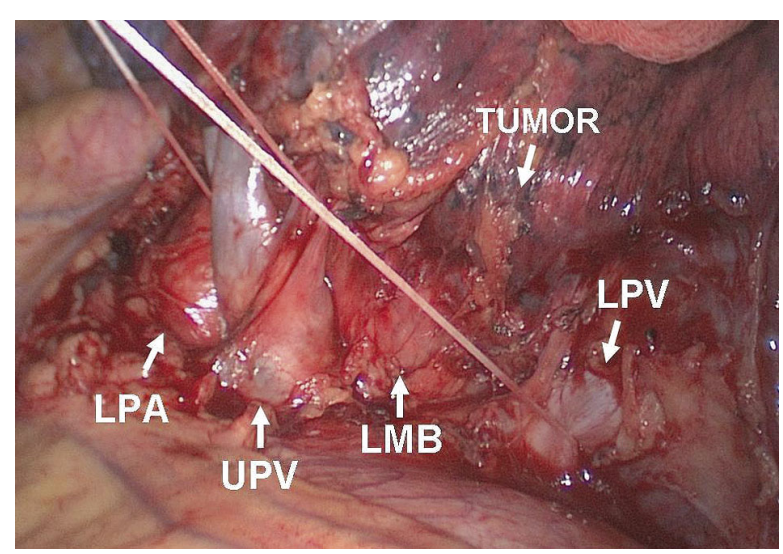

Figure 1. The left lung hilum

$\angle P A$ - left pulmonary artery, UPV - upper pulmonary vein, $L P V$ - lower pulmonary vein, $L M B$ - left main bronchus

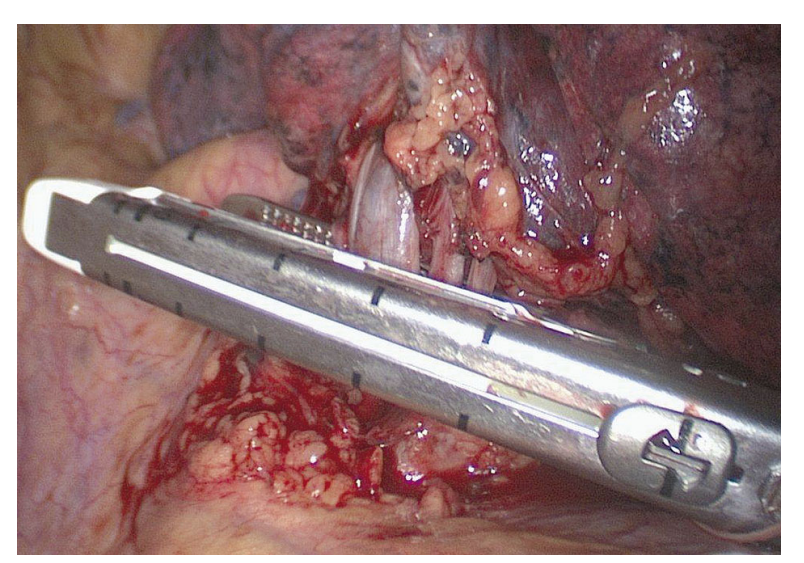

Figure 2. Endostapler on the left upper pulmonary vein ried out and the histopathological diagnosis of squamous cell carcinoma was made. Standard blood and coagulation tests were within the usual range. There were no pathological changes in abdominal ultrasound, ECG and echocardiography. Pulmonary function tests showed forced expiratory volume in $1 \mathrm{~s}$ $\left(\mathrm{FEV}_{1}\right)$ of 1.72 I (56.7\% of predicted value), forced vital capacity (FVC) 3.33 I (84.2\% of predicted value) and Tiffeneau index of $67.2 \%$. These results were considered as predicted postoperative values because of the almost complete obstruction of the left main bronchus and were acceptable for pneumonectomy. The patient was clinically staged as IB (T2aNOMO) and was qualified for VATS left pneumonectomy.

\section{Surgical technique}

Surgery was performed under combined general anesthesia with thoracic epidural analgesia. The patient was intubated with a right double-lumen endotracheal tube which allowed one lung ventilation during surgery. The patient was placed in the lateral decubitus position with flexion of the operating table at the level of the tip of the scapula to widen the intercostal spaces. Ports were placed in the same way as for VATS lobectomy: the first in the $7^{\text {th }}$ intercostal space in the anterior axillary line and the second in the $8^{\text {th }}$ intercostal space in the posterior axillary line. A $5 \mathrm{~cm}$ long utility incision was made at the level of the $4^{\text {th }}$ intercostal space anteriorly to the latissimus dorsi muscle. No rib spreader was used and the whole procedure was controlled on the monitor. The thoracoscope was introduced into the pleural cavity through the anterior port. Due to severe obstruction of the left main bronchus the lung was hyperinflated at the beginning of the procedure. It took some time of lung compression and bronchial suction to deflate the lung. After this maneuver the hilum could be well visualized. At first the pulmonary ligament was freed and the posterior mediastinal pleura of the hilum was dissected by electrocautery. Next the superior and inferior pulmonary veins and pulmonary artery were assessed and no neoplastic infiltration of the vessels in the hilum was found (Figure 1). The tumor infiltrating the main bronchus was revealed posterior to the upper pulmonary vein. Dense adhesions between the tumor and the posterior wall of the upper pulmonary vein were noticed and required careful preparation. After the dissection of the adhesions the vein was stapled and cut off 
with a vascular (white cartridge) $45 \mathrm{~mm}$ long endostapler (EndoGIA Ultra, Covidien, Mansfield, USA) (Figure 2). In order to achieve proper visualization of the right main pulmonary artery the mediastinal pleura in the aorto-pulmonary window was widely opened and the lymph nodes of stations 5 and 6 were removed. Next the dissection of the main left pulmonary artery was completed. A Satinsky clamp was introduced through the utility incision between the right main pulmonary artery and the left main bronchus and a ligature was placed around the artery (Figure 3). The 45-mm long vascular endostapler was introduced through the posterior port and the main left pulmonary artery was dissected (Figure 4). Last, the lower pulmonary vein was stapled and dissected in the same fashion. Subsequently the lung and left

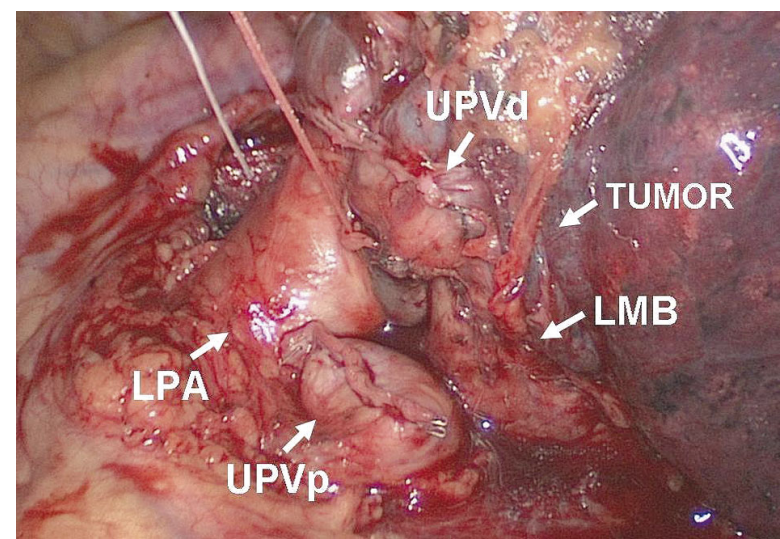

Figure 3. Left main pulmonary artery prepared for dissection

LPA - left pulmonary artery, UPVd - distal stump of the upper pulmonary vein, UPVp - proximal stump of the upper pulmonary vein, $L M B$ - left main bronchus

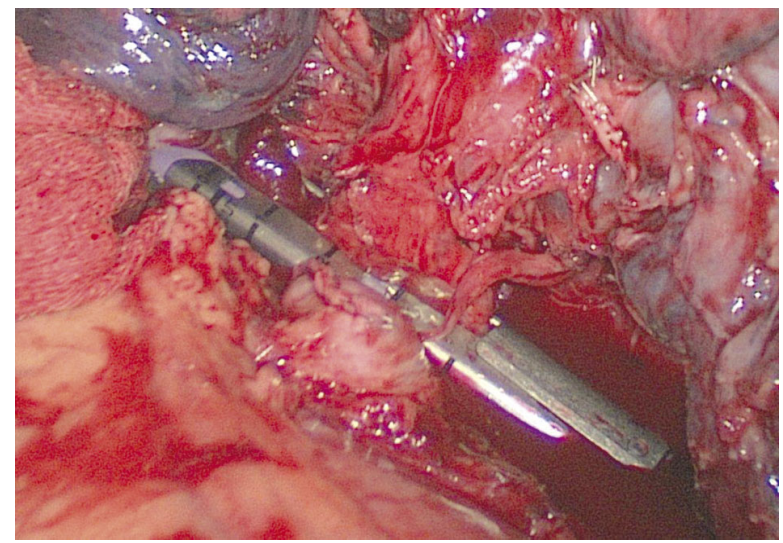

Figure 5. Endostapler on the left main bronchus main bronchus were lifted up and paraesophageal and subcarinal lymph nodes were removed. The left main bronchus was then dissected and cleared from the surrounding tissue up to the level of the carina and closed and stapled with 45-mm long green $(4.5 \mathrm{~mm})$ reload (Figure 5). The suture line was placed as proximally to the carina as possible to obtain a clear oncological margin. After bronchus dissection the lung was removed in the bag from the pleural cavity through the utility incision. The frozen section of the bronchial stump was clear. After lung removal the lymphadenectomy was completed and included lymph node stations 5, 6, 7, 8, 9 and 10. Peribronchial lymph nodes were removed with the lung. The bronchial stump was submerged under water to check for an air leak (Figure 6). Hemostasis was done

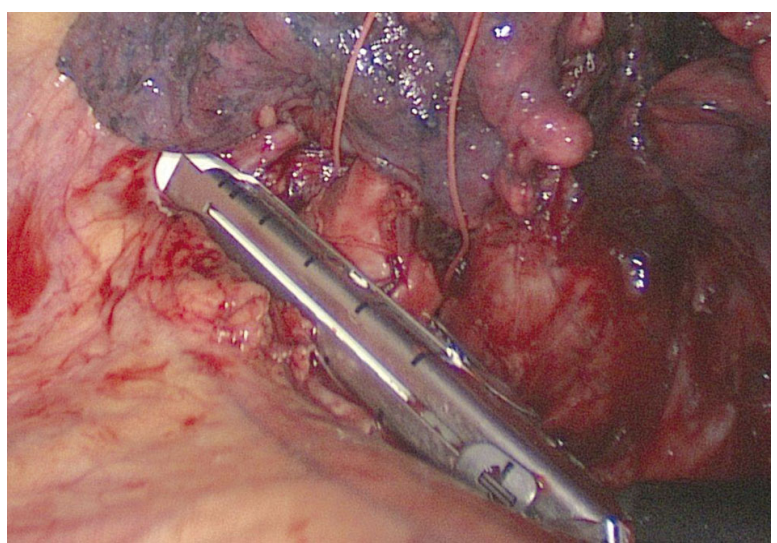

Figure 4. Endostapler on the left main pulmonary artery

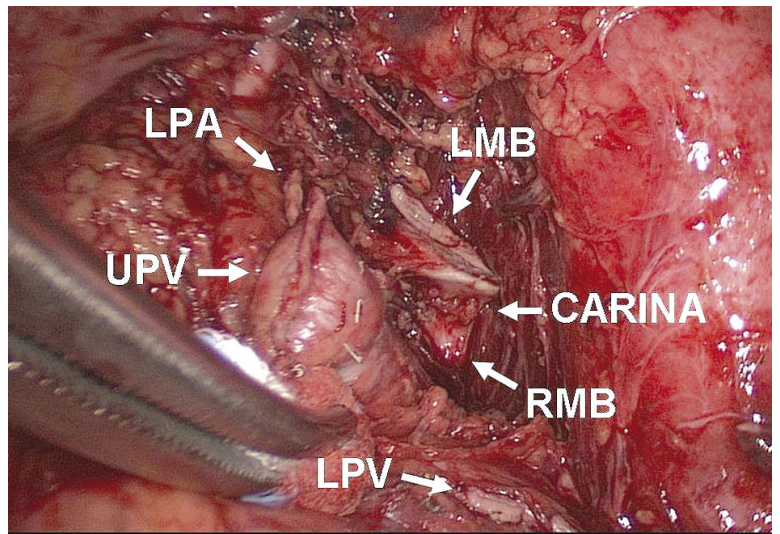

Figure 6. Left hilum after pneumonectomy and lymphadenectomy

LPA - stump of left main pulmonary artery, UPV - stump of the upper pulmonary vein, $L P V$ - lower pulmonary vein, $L M B$ - stump of the left main bronchus, $R M B$ - right main bronchus 
in the standard fashion. One $28 \mathrm{~F}$ chest tube was placed in the pleural cavity through the anterior port and connected to the underwater seal. The remaining port and utility incision were closed in a standard fashion without the intercostal suture. The patient was extubated in the operating theatre and transferred to the intensive care unit. Total surgery time was $130 \mathrm{~min}$ and blood loss was $150 \mathrm{ml}$. The postoperative course was complicated by atrial fibrillation on the $2^{\text {nd }}$ day after surgery which resolved after intravenous infusion of amiodarone. The chest tube was removed on the $2^{\text {nd }}$ post-operative day. Bronchofiberoscopic examination performed on the $7^{\text {th }}$ postoperative day showed proper healing of the bronchial stump and the patient was discharged home on the $8^{\text {th }}$ postoperative day. The final histopathological examination confirmed squamous cell lung cancer without lymph node involvement T2aNOMO stage IB.

\section{Discussion}

Pneumonectomy is performed mostly in patients with centrally located and advanced tumors. It is the most disabling type of anatomical resection and should be avoided if possible because of its pathophysiological consequences. The possibility of performing bronchial or vascular sleeve lobectomy should always be considered. Thorough preoperative evaluation of the CT scan and bronchoscopy is essential in this group of patients. In centrally located tumors thoracotomy still remains the best surgical approach for assessment of the appropriate type of resection.

Minimally invasive VATS pneumonectomy can be undertaken in patients with centrally located tumors without extended invasion of the large pulmonary vessels, chest wall, pericardium, mediastinal structures or the proximal part of the main bronchus and when sleeve resection is not feasible (T2). Another indication for VATS pneumonectomy can be peripherally located tumor with infiltration of all the lobes of the lung.

In the case of the patient described above, the tumor was small and invaded the distal part of the left main bronchus and both lobar bronchi, which precluded sleeve lobectomy. On the CT scan there was no invasion of adjacent structures $[6,7]$.

The pulmonary vessels and bronchus during thoracoscopy were managed in a similar order to open pneumonectomy. All structures of the hilum were closed with endostaplers [8].
Video-assisted thoracic surgery pneumonectomy is technically a very demanding procedure for several reasons. One of the intraoperative obstacles to deal with may be the difficulty with lung deflation caused by obstruction of the main bronchus despite a properly placed double lumen tracheal tube confirmed by intraoperative bronchoscopy. We observed such a problem in our patient. Taking some time to compress the lung may be necessary to deflate the lung and obtain enough empty pleural cavity space to safely perform the surgery. Another scenario can be massive inflammatory infiltration of the atelectatic lung, which makes the lung maneuvers much more difficult.

Another very important consideration is higher risk of injury of the pulmonary vessels during dissection caused by proximity of the tumor in the hilum. Lower mobility of hilar structures and resulting higher tension on the pulmonary artery increases the risk of rupture of the artery during dissection and introduction of the stapling device. Appropriate safety measures have to be taken to prevent and to prepare for such a situation.

The next matter to consider is management of the bronchus. Macroscopic assessment of the extent of the neoplastic invasion during surgery is much more limited in VATS compared to thoracotomy mostly because of lack of palpation. It makes preoperative bronchoscopic assessment performed by an experienced surgeon critically important. Microscopic examination of the bronchial margin is mandatory, and if the bronchial margin is found positive (R1) surgery should be extended. Conversion to thoracotomy and shortening of the bronchial stump or resection of the carina may be required.

In order to minimize the risk of bronchopleural fistula the endostapler should be closed slowly and gradually to allow proper approximation and suturing of the stiff cartilaginous wall of the main bronchus. Bronchial stump reinforcement with pedicled intercostal muscle flap, pericardial fat or mediastinal pleura is potentially possible but difficult and usually not done.

The last part of the surgery is mediastinal lymph node dissection. Visualization of the mediastinum after removal of the whole lung is much better than after lobectomy and the radical lymphadenectomy is much easier to perform.

In conclusion, VATS pneumonectomy should be performed only in centers with extensive experience 
in minimally invasive VATS lobectomy. Very limited indications for VATS pneumonectomy are the reason why the frequency of this type of procedure remains low. However, if the patients fulfill the VATS pneumonectomy inclusion criteria they may gain from all the advantages of minimally invasive techniques.

\section{References}

1. Downey RJ, Cheng D, Kernstine K, et al. Video-assisted thoracic surgery for lung cancer resection a consensus statement of the International Society of Minimally Invasive Cardiothoracic Surgery (ISMICS) 2007.

2. McKenna RJ, Houck W, Fuller CB. Video-assisted thoracic surgery lobectomy: experience with 1100 cases. Ann Thorac Surg 2006; 81: 421-6.

3. Swanson SJ, Herndon JE, D’Amico TA, et al. Video-assisted thoracic surgery (VATS) lobectomy. Report of CALGB 39802: a prospective, multi-institutional feasibility study. J Clin Oncol 2007; 25: 4993-7.

4. Sahai RK, Nwogu CE, Yendamuri S, et al. Is thoracoscopic pneumonectomy safe? Ann Thorac Surg 2009; 88: 1086-92.

5. Nwogu CE, Yendamuri S, Demmy TL. Does thoracoscopic pneumonectomy for lung cancer affect survival? Ann Thorac Surg 2010; 89: 2102-6.

6. Conlan AA, Sandor A. Thoracoscopic pneumonectomy: indications and technical considerations. J Thorac Cardiovasc Surg 2003; 126: 2083.

7. Roviaro G, Varoli F, Vergani C, et al. Techniques of pneumonectomy: video-assisted thoracic surgery pneumonectomy. Chest Surg Clin N Am 1999; 9: 419.

8. Walker WS, Carnochan FM, Mattar S. Video-assisted thoracoscopic pneumonectomy. Br J Surg 1994; 81: 81-2. 